\title{
PURIFICAÇÃO E CARACTERIZAÇÃO DE UMA GENTIOEXAOSE OBTIDA DE BOTRIOSFERANA POR HIDRÓLISE ÁCIDA PARCIAL
}

Iara Ribeiro Silva, Nilson Kobori Monteiro, Paula Felippe Martinez, Nataly Lino Izeli, Ana Flora Dalberto Vasconcelos, Marilsa de Stefani Cardoso e Maria de Lourdes Corradi da Silva*

Departamento de Física, Química e Biologia, Faculdade de Ciências e Tecnologia, Universidade Estadual Paulista Júlio de Mesquita Filho, CP 467, 19060-900 Presidente Prudente - SP, Brasil

Aneli de Melo Barbosa

Departamento de Bioquímica e Biotecnologia, Centro de Ciências Exatas, Universidade Estadual de Londrina, CP 6001, 86051-990 Londrina - PR, Brasil

Robert F. H. Dekker

Universidad de Castilla-La Mancha - IRICA, 13071 Ciudad Real, España

Gil Valdo José da Silva e Luís Alberto Beraldo de Moraes

Departamento de Química, Faculdade de Filosofia, Ciências e Letras de Ribeirão Preto, Universidade de São Paulo, 14040-901

Ribeirão Preto - SP, Brasil

Recebido em 27/2/07; aceito em 11/10/07; publicado na web em 9/4/08

\begin{abstract}
PURIFICATION AND CHARACTERIZATION OF A GENTIOHEXAOSE OBTAINED FROM BOTRYOSPHAERAN BY PARTIAL ACID HYDROLYSIS. A hexa-oligosaccharide was obtained by partial acid hydrolysis from botryosphaeran, an exopolysaccharide (EPS) $\beta(1 \rightarrow 3 ; 1 \rightarrow 6)$-D-glucan type, produced by the ascomyceteous fungus Botryosphaeria rhodina. The oligosaccharide was purified by gel filtration and charcoal-Celite column chromatography and the analysis was followed by HPAEC/ PAD. The structure was determined by NMR spectroscopy and mass spectrometry, which showed that the oligosaccharide consists of six $\beta$-D-glucopiranosyl units $O-6$ substituted (gentiohexaose).
\end{abstract}

Keywords: oligosaccharide; partial acid hydrolysis; $\beta$-glucan.

\section{INTRODUÇÃO}

Botriosferana é um exopolissacarídeo (EPS) do tipo $\beta$-Dglucana, produzido pelo fungo ascomiceto Botryosphaeria rhodina MAMB-05, quando cultivado em glucose ${ }^{1}$ como fonte única de carbono. O polímero foi caracterizado como uma $\beta(1 \rightarrow 3 ; 1 \rightarrow 6)$-Dglucana com aproximadamente $22 \%$ de ramificações, as quais consistem de resíduos glucosídicos e gentiobiosídicos, vinculados à cadeia principal por ligações tipo $\beta(1 \rightarrow 6){ }^{2}$

O botriosferana foi também obtido quando cultivado em outras fontes de carbono (frutose, galactose, manose, lactose, sacarose, melaço de cana-de-açúcar), com a maior produção ocorrendo em sacarose. ${ }^{3} \mathrm{O}$ EPS produzido em frutose apresentou mais ramificações $(31 \%)$ que aquele obtido em sacarose $(21 \%)$, o qual foi semelhante ao EPS produzido em glucose. ${ }^{4}$

Algumas $\beta$-glucanas não celulósicas têm sido avaliadas quanto ao seu potencial biológico e podem apresentar atividade anti-tumor, anti-inflamatória e imunomoduladora. ${ }^{5-7}$ Estudos recentes demonstraram que o botriosferana não é mutagênico e possui em torno de $82 \%$ de atividade antimutagênica. ${ }^{8}$ Oligossacarídeos pequenos também podem influenciar positivamente o sistema imune. ${ }^{9}$

Existem peculiaridades estruturais das $\beta$-glucanas responsáveis pela baixa solubilidade em água e formação de soluções viscosas, que dificultam a aplicação clínica destes biopolímeros. A fragmentação destas moléculas pela utilização de enzimas resolve o problema da solubilidade e certos fragmentos podem reter a atividade biológica. ${ }^{10} \mathrm{O}$ botriosferana foi utilizado como substrato para a

*e-mail: corradi@fct.unesp.br produção de $\beta$-1,3-glucanases por Botryosphaeria rhodina e Trichoderma harzianum Rifai. A forma de atuação de ambas as $\beta$ glucanases foi diferente, de acordo com os produtos de hidrólise..$^{10,11}$

Uma outra forma de obtenção de oligossacarídeos pode ser a hidrólise ácida parcial de $\beta$-glucanas. O tipo de ácido, sua concentração e as condições de hidrólise (tempo e temperatura) devem ser padronizados para se obter os oligossacarídeos de interesse. ${ }^{12}$

Recentemente verificou-se que muitos oligossacarídeos, principalmente aqueles com seis ou mais unidades monossacarídicas, conservam ou até mesmo potencializam as propriedades biológicas de seus polímeros. Uma possível explicação para essa constatação é a melhor solubilidade dos oligossacarídeos quando comparados às suas moléculas nativas, bem como ao tamanho dos oligossacarídeos e a manutenção da conformação da molécula original. Novas funções fisiológicas importantes que beneficiam a saúde humana têm sido descritas para os oligossacarídeos, incluindo-se um efeito imunomodulador na prevenção de alergia e de câncer. ${ }^{13,14}$

O presente trabalho descreve, pela primeira vez, a purificação e caracterização química de um hexaoligossacarídeo obtido por hidrólise ácida parcial da $\beta$-glucana produzida pelo fungo ascomiceto Botryosphaeria rhodina, denominado botriosferana.

\section{PARTE EXPERIMENTAL}

\section{Manutenção do Botryosphaeria rhodina e preparo do inóculo para a produção do botriosferana}

O fungo foi mantido em BDA (batata-dextrose-ágar) inclinado, a $4{ }^{\circ} \mathrm{C}$, com repiques trimestrais. 
Para o preparo do inóculo, o fungo foi transferido para o meio sólido de sais de Vogel ${ }^{15}$ contendo ágar a $20 \mathrm{~g} / \mathrm{L}(\mathrm{m} / \mathrm{v})$ e glucose $10 \mathrm{~g} / \mathrm{L}(\mathrm{m} / \mathrm{v})$. As placas foram incubadas durante 5 dias a $28{ }^{\circ} \mathrm{C} \mathrm{em}$ estufa bacteriológica. Pequenas porções de hifas foram transferidas para dois erlenmeyer $(125 \mathrm{~mL})$ contendo $25 \mathrm{~mL}$ de meio de Vogel ${ }^{15}$ e $5 \mathrm{~g} / \mathrm{L}(\mathrm{m} / \mathrm{v})$ de glucose. Os cultivos foram mantidos sob agitação constante a $180 \mathrm{rpm}$ por $48 \mathrm{~h}$, a $28{ }^{\circ} \mathrm{C}$. Em seguida, os micélios foram tratados em um homogeneizador de células, à velocidade máxima, durante $30 \mathrm{~s}$. O homogeinato de células foi centrifugado durante $10 \mathrm{~min}$ a $7000 \mathrm{xg}$, os sobrenadantes decantados e o precipitado foi ressuspendido em uma solução de salina fisiológica estéril, até se obter um valor de absorbância entre 0,4 e 0,5 a $400 \mathrm{~nm}$.

\section{Produção da $\beta$-glucana botriosferana}

Os cultivos foram desenvolvidos em frascos de erlenmeyer de $2000 \mathrm{~mL}$ contendo $400 \mathrm{~mL}$ de meio de $\operatorname{Vogel}^{15}$ e $50 \mathrm{~g} / \mathrm{L}(\mathrm{m} / \mathrm{v})$ de glucose. Cada frasco foi inoculado com $16 \mathrm{~mL}$ da solução do homogeinato de células descrito no item anterior. Os frascos foram agitados a $180 \mathrm{rpm}$, durante $72 \mathrm{~h} \mathrm{a} 28^{\circ} \mathrm{C}$. Os cultivos foram interrompidos por centrifugação $(7000 \mathrm{xg} / 10 \mathrm{~min})$ a $4{ }^{\circ} \mathrm{C}$. Os sobrenadantes foram coletados em banho de gelo e dialisados (MWCO $12000 \mathrm{Da}$ ), exaustivamente, contra água destilada. Em seguida, as frações retidas no tubo de diálise foram tratadas com três volumes de etanol absoluto, previamente gelado a $4{ }^{\circ} \mathrm{C}$, para precipitar o EPS botriosferana. Depois de mantido por, no mínimo, $2 \mathrm{~h}$ em câmara fria a $4{ }^{\circ} \mathrm{C}$, o EPS foi ressolubilizado em água destilada e novamente dialisado com água destilada para eliminar todo o etanol e, em seguida, liofilizado. Estas preparações foram utilizadas para os procedimentos de quantificação de açúcares totais $^{16}$ e redutores..$^{17,18}$

\section{Hidrólise ácida parcial do botriosferana ${ }^{19}$}

À massa total de exopolissacarídeo $(240 \mathrm{mg})$ foram adicionados $240 \mathrm{~mL}$ de TFA $0,2 \mathrm{M}$, em tubo próprio para hidrólise. A solução resultante, viscosa, sofreu curtos períodos de agitação mecânica e então foi colocada em estufa a $100{ }^{\circ} \mathrm{C}$ por $240 \mathrm{~min}$. Periodicamente, o tubo era retirado da estufa e agitado para melhor solubilização da amostra. Decorrido o tempo, o TFA foi removido por evaporação, sob baixa pressão, a amostra foi lavada com porções de água destilada, seguida de evaporação até eliminação total do ácido. $\mathrm{O}$ hidrolisado parcial resultante foi solubilizado em água e o material insolúvel foi centrifugado a $7500 \mathrm{xg}$ por $30 \mathrm{~min}$. O sobrenadante, ainda turvo, foi tratado com três volumes de etanol absoluto gelado e mantido a $4{ }^{\circ} \mathrm{C}$, durante a noite. O sobrenadante etanólico, que continha os oligossacarídeos e o precipitado, que continha o polissacarídeo residual, foi separado por centrifugação à $7500 \mathrm{xg} / 30 \mathrm{~min}$. Ambos foram evaporados até secura, para eliminar todo o álcool e, em seguida, solubilizados em água deionizada. Posteriormente, alíquotas foram retiradas para serem quantificados os carboidratos totais e então analisados por HPAEC/PAD (high performance anionic exchange chromatography-pulsed amperometric detector).

\section{Purificação por métodos cromatográficos dos fragmentos oligossacarídicos obtidos por hidrólise ácida parcial do botriosferana}

\section{Cromatografia de gel permeação em Sephadex G-15}

$\mathrm{O}$ hidrolisado foi solubilizado em água destilada e aplicado em uma coluna de vidro $(103,0 \times 0,8 \mathrm{~cm})$ preenchida com gel Sephadex G-15. Água destilada foi utilizada como eluente e o fluxo de opera- ção da coluna foi de $0,8 \mathrm{~mL} / \mathrm{min}$. Frações de 1,4 mL foram coletadas e alíquotas de cada uma das frações foram analisadas para detectar açúcares totais pelo método do fenol-ácido sulfúrico. ${ }^{16}$

Após a determinação do perfil de eluição, as frações referentes aos picos distintos de açúcares foram reunidas e liofilizadas. ${ }^{20}$

\section{Cromatografia em coluna de carvão-Celite ${ }^{21,22}$}

Partes iguais de carvão ativado, pó fino, (marca Riedel) e Celite foram misturadas com água. A mistura resultante, na forma de "papa", foi adicionada à coluna de vidro. Para a remoção de traços de metais a coluna empacotada foi lavada, lentamente, com $\mathrm{HCl}$ concentrado e, em seguida, com água até $\mathrm{pH}$ neutro.

Duas colunas de carvão-Celite foram utilizadas para separar o material de interesse, obtido previamente pela cromatografia de gel permeação (pico A). A primeira coluna de carvão-Celite $(28 \times 2,5$ $\mathrm{cm})$ foi eluída com um gradiente descontínuo de etanol-água (v/v) 20, 25 e $30 \%$, fluxo de operação de $0,34 \mathrm{~mL} / \mathrm{min}$ e frações de 1,4 $\mathrm{mL}$; a segunda coluna $(16,7 \times 1,2 \mathrm{~cm})$ foi eluída com etanol-água (v/v) 26, 28 e $30 \%$, fluxo de operação $0,50 \mathrm{~mL} / \mathrm{min}$ e frações de 2,0 mL, objetivando purificar a fração de interesse (etanol-água $30 \%$ ), separada pela primeira coluna.

\section{Análise dos oligossacarídeos ${ }^{23}$}

Todas as frações procedentes das cromatografias de gel permeação e carvão-Celite foram analisadas por cromatografia líquida de alta pressão, em sistema Dionex DX 500 com detector de amperometria pulsada (PAD). Os cromatogramas foram registrados em um integrador modelo 4600.

Os oligossacarídeos foram separados com um gradiente de acetato de sódio (3 a 40\%), em uma coluna analítica CarboPac PA100 (4 x $250 \mathrm{~mm}$ ) equipada com uma guarda coluna PA100, à velocidade de fluxo de $1,0 \mathrm{~mL} / \mathrm{min}$. As condições de eluição foram produzidas com acetato de sódio $500 \mathrm{mM}$ (eluente 1) e $\mathrm{NaOH} 100 \mathrm{mM}$ (eluente 2), este último preparado a partir de solução de $\mathrm{NaOH} 50 \%$.

Após cada corrida cromatográfica (70 $\mathrm{min}$ ) a coluna foi regenerada, utilizando-se $100 \%$ do eluente 1 (10 min) e então, antes de nova injeção da amostra, ${ }^{24,25}$ foi equilibrada por 15 min nas condições iniciais da corrida ( $3 \%$ do eluente 1$)$.

\section{Análise de ressonância magnética nuclear de próton $\left(\mathrm{RMN}{ }^{1} \mathrm{H}\right)$ e de carbono treze $\left(\mathrm{RMN}{ }^{13} \mathrm{C}\right)$}

Previamente à análise, o oligossacarídeo em estudo foi dissolvido em óxido de deutério $\left(99,9\right.$ atom\% $\left.{ }^{2} \mathrm{H}\right)$ em uma concentração de $1,0 \mathrm{mg} / \mathrm{mL}$, sendo submetido, durante a análise, a uma temperatura de $300{ }^{\circ} \mathrm{K}$.

Os espectros de alta resolução foram obtidos em um espectrômetro Bruker DRX 500 (freq. $1 \mathrm{H}=500.13 \mathrm{MHz}$ ), equipado com uma sonda de $2,5 \mathrm{~mm}$ com gradiente de campo no eixo z. Os deslocamentos químicos $(\delta)$ foram expressos em ppm, relativos ao padrão interno acetona $\left(\delta{ }^{1} \mathrm{H}: 2,22 \mathrm{ppm}\right.$ e $\left.\delta{ }^{13} \mathrm{C}: 30,2 \mathrm{ppm}\right)$. As análises por RMN envolveram técnicas bidimensionais: ${ }^{1} \mathrm{H}-{ }^{1} \mathrm{H}$ COSY (aquisição: 2048 x 256 pontos de dados, 32 transientes; largura: $4921 \mathrm{~Hz}$ em ambas dimensões; espectro processado: 1024 x 1024 pontos), ${ }^{1} \mathrm{H}-{ }^{13} \mathrm{C}$ HMQC (aquisição: 1024 x 256 pontos de dados, 120 transientes; largura: 4921 Hz em F2 e 20833 Hz em F1; espectro processado: 1024 x 1024 pontos).

\section{Espectrometria de massa}

As análises por espectrometria de massas foram realizadas utilizando-se um espectrômetro de massa Q-Tof (Micromass, 
Manchester, UK) do tipo híbrido quadrupole time of flight (TFO) de alta resolução (7.000) e sensibilidade (5 ppm), equipado com uma fonte de ionização por electrospray (ESI). As condições de trabalho para o ESI positivo e negativo foram as seguintes: gás de solvatação (nitrogênio) a $250{ }^{\circ} \mathrm{C}$; capilaridade $3,2 \mathrm{kV}$ e cone de voltagem $25 \mathrm{kV}$. Os espectros de massas de MS/MS foram adquiridos através da seleção de massa em Q1, seguida da dissociação induzida por colisão com argônio em q2 e a análise de massas dos fragmentos no TOF. $\mathrm{O}$ extrato bruto foi dissolvido em uma mistura de $\mathrm{MeOH} / \mathrm{D}_{2} \mathrm{O}$ 2:1 (v/v) e acetato de amônio $10 \mathrm{mM}$ para análises em ambos os modos de íons positivo e negativo. A amostra foi introduzida na fonte de electrospray com uma bomba de seringa com fluxo de $10 \mu \mathrm{L} / \mathrm{min}$.

\section{RESULTADOS E DISCUSSÃO}

O botriosferana é um exopolissacarídeo produzido pelo Botryosphaeria rhodina cuja estrutura já foi caracterizada por Barbosa e colaboradores ${ }^{2}$ como uma glucana constituída de uma cadeia principal $\beta(1 \rightarrow 3)$ com ramificações no carbono 6 . O grande inconveniente e limitação na aplicação deste EPS está em sua baixa solubilidade em água ( $0,3 \mathrm{~g} \%)$; entretanto, essa situação pode ser contornada se a molécula original for degradada a fragmentos menores, que certamente serão solúveis em água. Neste trabalho, foi determinada uma condição de hidrólise para obtenção de oligossacarídeos a partir do botriosferana.

Há poucos artigos na literatura sobre a obtenção de oligossacarídeos por hidrólise ácida parcial de polímeros em comparação àqueles que tratam de hidrólise enzimática, esta última altamente específica quanto às ligações glicosídicas a serem rompidas. ${ }^{26}$ Porém, a grande vantagem da hidrólise ácida está na possibilidade de ser realizada em temperaturas elevadas promovendo maior fluidez de soluções viscosas. Geralmente, após seleção do melhor ácido bem como de sua concentração, tempo e temperatura de reação, através de curvas de hidrólise apropriadas, é possível gerar di-, tri- e oligossacarídeos maiores. ${ }^{19,27}$

Segundo Bao e colaboradores, ${ }^{19}$ a fragmentação parcial de um polímero original pode ser realizada por hidrólise com ácido trifluoracético (TFA), utilizado neste trabalho, que apresenta como vantagem sobre os demais ácidos a sua remoção por evaporação sob pressão reduzida. $\mathrm{O}$ desenvolvimento de uma cinética de tempo estabeleceu 240 min como ideal para obtenção de oligossacarídeos maiores. Oligossacarídeos com grau de polimerização entre 5 e 7 unidades mantêm a atividade biológica do polímero original, ${ }^{13,28}$ desde que a conformação da molécula seja preservada.

Após o tempo de reação, o hidrolisado foi analisado por cromatografia líquida de íons acoplada a um detector de amperometria pulsada (HPAEC/PAD) sendo caracterizado como uma mistura de oligossacarídeos (Figura 1) de diferentes massas moleculares, provenientes da fragmentação aleatória promovida pelo ácido.

Foram selecionados dois métodos cromatográficos para a separação dos oligossacarídeos, filtração em gel e carvão-Celite, à pressão normal.

O hidrolisado foi aplicado a uma coluna de filtração em gel Sephadex G-15 e o perfil de eluição dos oligossacarídeos foi acompanhado por HPAEC/PAD (Figura 2). O fragmento de menor massa molecular, com tempo de retenção semelhante à glucose, foi eluído no segundo pico (B), e os de maior massa molecular no primeiro pico (A). Apesar da cromatografia de gel permeação à pressão normal não ter sido eficiente na separação dos diferentes oligossacarídeos foi, entretanto, capaz de separar o componente monossacarídico, quantitativamente predominante no hidrolisado, das moléculas de maior massa. O pico A, contendo os fragmentos maiores, foi separado para dar sequiência ao estudo.

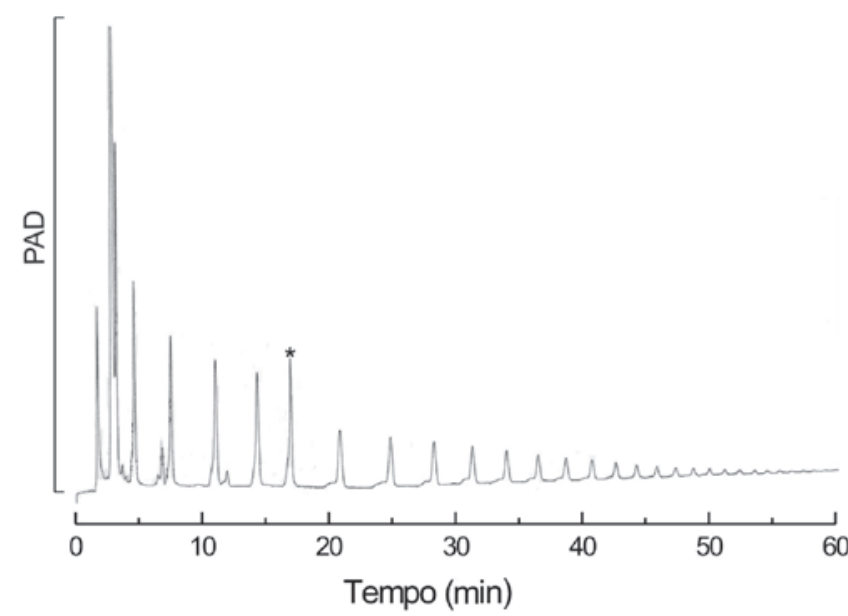

Figura 1. Análise por HPAEC/PAD do hidrolisado ácido parcial do botriosferana. Condições da hidrólise: TFA 0,2 M, $100{ }^{\circ} \mathrm{C}, 4 \mathrm{~h}$; coluna analítica CarboPac PA100; material aplicado: 1,1 $\mu \mathrm{g}$ de açúcares totais em $25 \mu \mathrm{L}$. * representa componente com $R_{T}=16,7 \mathrm{~min}$

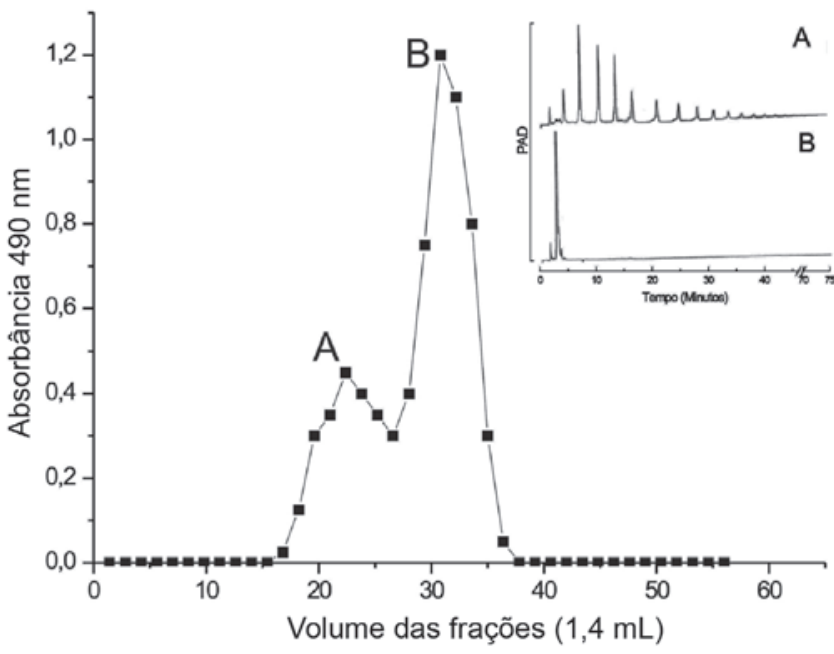

Figura 2. Cromatografia de gel permeação do hidrolisado ácido parcial do botriosferana em coluna de gel Sephadex G-15. Dimensões da coluna: $103 x$ 0,8 cm; eluente: água; fluxo: 0,8 mL/min; volume da fração: 1,4 mL. Picos Ae $B$ analisados por HPAEC/PAD em coluna analítica CarboPac PA 100

De acordo com Whistler e BeMiller, ${ }^{21}$ a cromatografia de adsorção em carvão-Celite pode ser utilizada com sucesso na separação de séries homólogas de oligossacarídeos geralmente pela utilização de um gradiente etanol-água. Corradi da Silva e colaboradores ${ }^{22}$ separaram, eficientemente, uma série homóloga de oligossacarídeos provenientes de um extrato metanol-água do líquen Parmotrema cetratum; posteriormente essas moléculas foram caracterizadas por RMN de próton e espectrometria de massa como di-, tri-, tetra- e penta-gentioligossacarídeos.

A solução aquosa de oligossacarídeos (pico A, Figura 2) foi aplicada a uma coluna de carvão-Celite (Figura 3-A) e eluída com um gradiente descontínuo de etanol-água (20, 25 e 30\%). O acompanhamento dos eluatos por HPAEC/PAD mostrou que a fração eluída com etanol-água 30\% (Figura 3-A ) era constituída por um material quase puro. Esta foi recromatografada em coluna semelhante (Figura 3-B) e um componente com tempo de retenção de 16,76 min foi eluído com etanol-água 28\% (Figura 3-B ), com rendimento de 5\% em relação ao total de oligossacarídeos $(39,0 \mathrm{mg})$ obtidos na hidrólise ácida parcial. 

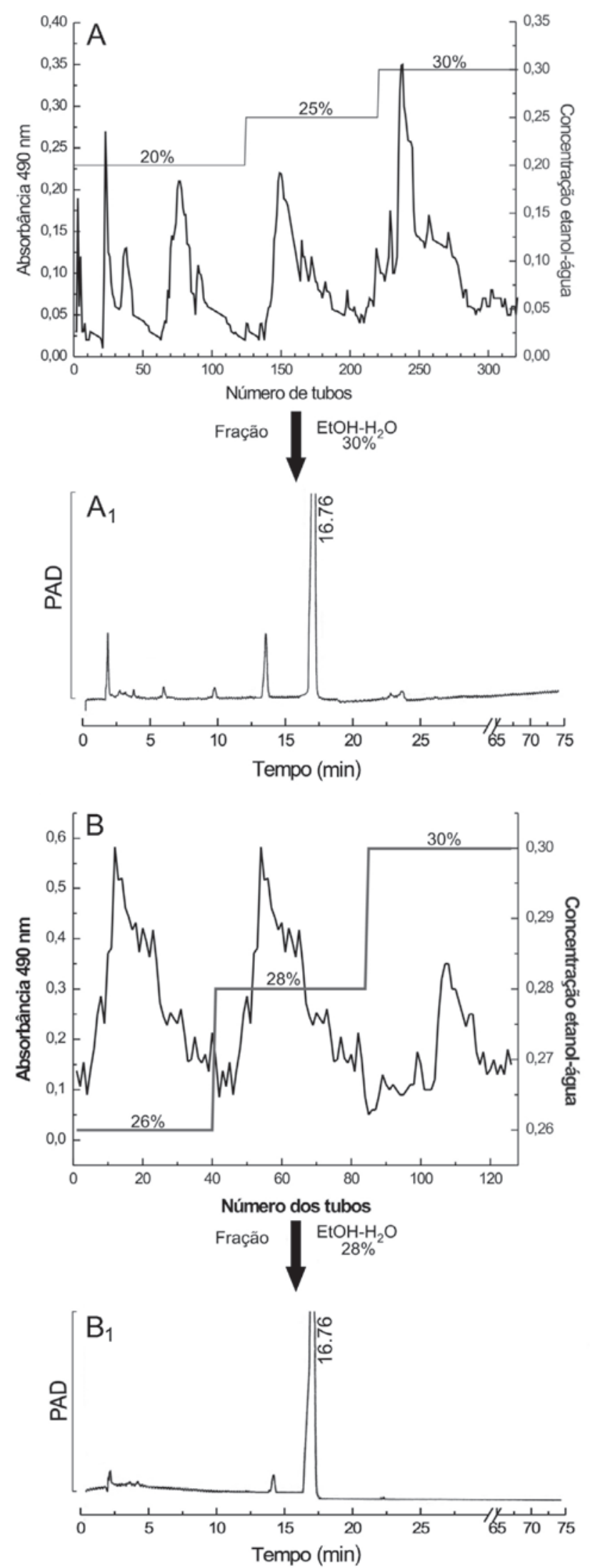

Figura 3. Cromatografia em carvão-Celite $(\boldsymbol{A})$ do primeiro pico proveniente da cromatografia de gel permeação, com respectiva análise por HPAEC/ PAD (fração eluída a $30 \%$ - $\boldsymbol{A}_{1}$ ). Cromatografia em carvão-Celite (B) da fração $\mathrm{EtOH}-\mathrm{H}_{2} \mathrm{O} 30 \%$ e respectiva análise por HPAEC/PAD (fração eluída a $\left.28 \%-B_{1}\right)$
Análises espectroscópicas de $\mathrm{RMN}{ }^{1} \mathrm{H}$ e $\mathrm{RMN}{ }^{13} \mathrm{C}$ foram realizadas para caracterização química do componente.

Comparando-se os espectros de $\mathrm{RMN}{ }^{13} \mathrm{C}$ do componente em estudo com dados da literatura, ${ }^{22,29}$ os deslocamentos químicos foram caracterizados como sendo de uma molécula constituída por unidades glucopiranosídicas $1 \rightarrow 6$ substituídas. A ausência de sinais na região entre 77,0 e 90,0 ppm é uma forte evidência de uma molécula 6- $O$-substituída.

$\mathrm{O}$ espectro de correlação heteronuclear ${ }^{1} \mathrm{H}-{ }^{13} \mathrm{C}$ HMQC (Figura $1 \mathrm{~S}-\mathrm{A}$, material suplementar) apresenta um sinal em $\delta_{\mathrm{H}} 4,42$ e $\delta_{\mathrm{C}}$ 103,47 atribuído à posição anomérica das unidades $\beta$-Dglucopiranosídicas não redutoras; observa-se também um sinal de menor intensidade em $\delta_{\mathrm{H}} 4,56$ e $\delta_{\mathrm{C}} 96,18$, correspondente à extremidade redutora da molécula na forma $\beta .{ }^{29} \mathrm{O}$ espectro de correlação homonuclear ${ }^{1} \mathrm{H}-{ }^{1} \mathrm{H}$ COSY (Figura 1S-B) mostra um acoplamento do próton em $\delta_{\mathrm{H}} 4,42(\mathrm{H}-1)$ com o próton em $\delta_{\mathrm{H}} 3,23$, o que indica que este último corresponde a H-2. A análise da correlação do COSY permite atribuir os sinais correspondentes aos demais hidrogênios $\left(\delta_{\mathrm{H}-3}: 3,39 ; \delta_{\mathrm{H}-4}: 3,38 ; \delta_{\mathrm{H}-5}: 3,53 ; \delta_{\mathrm{H}-6 \mathrm{a}}: 3,77\right.$ e $\delta_{\mathrm{H}-66}$ : 4,13). A análise conjunta dos espectros de HMQC (Figura 1S-A) e COSY (Figura 1S-B) permitiu fazer a atribuição de todos os ${ }^{1} \mathrm{H}$ e ${ }^{13} \mathrm{C}$ da molécula. A correspondência entre os espectros permite atribuir os sinais em $\delta_{\mathrm{C}} 73,43 ; 75,23 ; 69,69$ e 75,18, respectivamente, aos carbonos 2, 3, 4 e 5 das unidades glucosídicas. O sinal de ${ }^{13} \mathrm{C}$ em 69,06 ppm, correlacionado aos sinais de ${ }^{1} \mathrm{H}$ em 3,77 e 4,13 ppm, refere-se ao C-6 $O$-substituído. O componente C-6, com $\mathrm{OH}$ livre da extremidade terminal não redutora, aparece em 61,02 ppm e está relacionado aos ${ }^{1} \mathrm{H}$ em 3,63 e 3,80 ppm.

$\mathrm{O}$ espectro de massa do oligossacarídeo no modo negativo (Figura 2S-A) mostrou um cluster de sinais centrados em $\mathrm{m} / \mathrm{z}$, 991,7 (M$\mathrm{H})^{-}$, que foi atribuído a um oligossacarídeo com seis unidades glucopiranosídicas. Esses picos correspondem aos diversos níveis de troca isotópica devido à dissolução da amostra em $\mathrm{D}_{2} \mathrm{O}$. $\mathrm{O}$ espectro de massas no modo positivo (Figura 2S-B) apresentou dois cluster de sinais de $m / z$ 1015,7 e 1031,7, os quais foram atribuídos à cationização com sódio $(\mathrm{M}+\mathrm{Na})^{+}$e potássio $(\mathrm{M}+\mathrm{K})^{+}$, respectivamente. $\mathrm{O}$ espectro de fragmentação (Figura $2 \mathrm{~S}-\mathrm{C}$ ) mostrando perdas sucessivas de unidades de glucose (162 uma) é consistente com a massa molecular do oligossacarídeo contendo seis unidades glucosídicas.

\section{CONCLUSÕES}

A partir das análises efetuadas neste trabalho, pode-se concluir que o oligossacarídeo obtido por hidrólise ácida parcial do botriosferana e purificado pelos métodos cromatográficos descritos, trata-se de uma molécula constituída, exclusivamente, por unidades glucopiranosídicas $\beta(1 \rightarrow 6)$, cuja estrutura encontra-se representada abaixo.

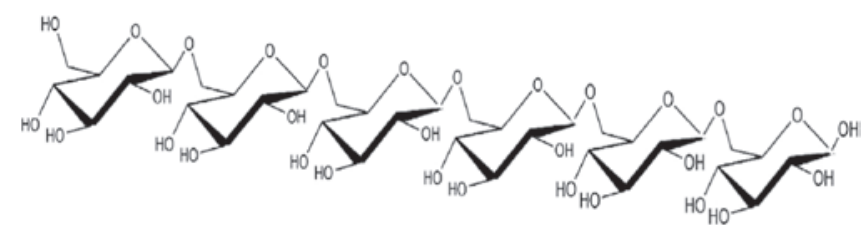

Estrutura 1.

Estudos posteriores sobre o potencial biológico deste hexaoligossacarídeo poderão ser realizados, considerando-se o aumento do grau de solubilidade dessa molécula quando comparado ao do polímero original, botriosferana. 


\section{MATERIAL SUPLEMENTAR}

As figuras relativas às análises de ressonância de carbono e próton (1S-A e 1S-B) e espectrometria de massa (2S-A, 2S-B e 2SC) do oligossacarídeo purificado encontram-se no material suplementar disponível gratuitamente em http://quimicanova.sbq.org.br, na forma de arquivo pdf, com acesso livre.

\section{AGRADECIMENTOS}

À FAPESP (processo $n^{\circ}$ 02/13888-5), CNPq e Fundação Araucária (Projeto $\mathrm{n}^{\circ}$ 5777).

\section{REFERÊNCIAS}

1. Dekker, R. F. H.; Barbosa, A. M.; Enzyme Microb. Technol. 2001, $28,81$.

2. Barbosa, A. M.; Steluti, R. M.; Dekker, R. F. H.; Cardoso, M. S.; Corradi da Silva, M. L.; Carbohydr. Res. 2003, 338, 1691

3. Steluti, R. M.; Giese, E. C.; Pigatto, M. M.; Sumiya, A. F. G.; Covizzi, L. G.; Job, A. E.; Cardoso, M. S.; Corradi da Silva, M. L.; Dekker, R. F. H.; Barbosa, A. M.; J. Basic Microbiol. 2004, 44, 480.

4. Corradi da Silva, M. L.; Izeli, N. L.; Martinez, P. F.; Silva, I. R.; Constantino, C. J. L.; Cardoso, M. S.; Barbosa, A. M.; Dekker, R. F. H.; da Silva, G. V. J.; Carbohydr. Polym. 2005, 61, 10.

5. Wasser, S. P.; Appl. Microbiol. Biotechnol. 2002, 60, 258.

6. Paulsen, B. S.; Phytochem. Rev. 2002, 1, 379.

7. Corradi da Silva, M. L.; Martinez, P. F.; Izeli, N. L.; Silva, I. R.; Vasconcelos, A. F. D.; Cardoso, M. S.; Steluti, R. M.; Giese, E. C.; Barbosa, A. M.; Quim. Nova 2006, 29, 85.

8. Miranda, C. C. B. O.; Dissertação de Mestrado, Universidade Estadual de Londrina, Brasil, 2006
9. Klarzynski, O.; Plesse, B.; Joubert, J-M.; Yvin, J-C.; Koop, M.; Kloareg, B.; Fritig, B.; Plant Physiol. 2000, 124, 1027.

10. Giese, E. C.; Covizzi, L. G.; Borsato, D.; Dekker, R. F. H.; Corradi da Silva, M. L.; Barbosa, A. M.; Process Biochem. 2005, 40, 3783.

11. Giese, E. C.; Covizzi, L. G.; Dekker, R. F. H.; Monteiro, N. K.; Corradi da Silva, M. L.; Barbosa, A. M.; Process Biochem. 2006, 41, 1265.

12. Johansson, L.; Virkki, L.; Anttila, H.; Esselström, H.; Tuomainen, P.; SontagStrohm, T.; Food Chem. 2006, 97, 71.

13. Bland, E. J.; Keshavarz, T.; Bucke, C.; Carbohydr. Res. 2004, 339, 1673.

14. Hirayama, M.; Pure Appl. Chem. 2002, 74, 1271.

15. Vogel, H. J.; Genet. Bull. 1956, 13, 42.

16. Dubois, M.; Gilles, K. A.; Hamilton, J. K.; Reber, P. A.; Smith, F.; Anal. Chem. 1956, 28, 350.

17. Nelson, N. A.; J. Biol. Chem. 1944, 153, 376.

18. Somogyi, M. A.; J. Biol. Chem. 1945, 160, 61.

19. Bao, X-F.; Zhen, Y.; Ruan, L.; Fang, J-N.; Chem. Pharm. Bull. 2002, 50, 623.

20. Gutierrez, A.; Prieto, A.; Martinez; A. T.; Carbohydr. Res. 1996, 281, 143.

21. Whistler, R. L.; BeMiller, J. N.; Meth. Carbohydr. Chem. 1962, 1, 47.

22. Corradi da Silva, M. L.; Iacomini, M.; Jablonski, E.; Gorin, P. A.; Phytochemistry 1993, 35, 547.

23. Oliveira, P. F.; Doná, F.; Marcelli, M.; Cardoso, M. S.; Corradi da Silva, M. L.; Eclética Quim. 2002, 27, 211.

24. Rice, K. G.; Corradi da Silva, M. L.; J. Chromatogr., A 1996, 720, 235

25. Elifio, S. L.; Corradi da Silva, M. L.; Iacomini, M.; Gorin, P. A. J.; New Phytol. 2000, 145, 327.

26. Schmid, F.; Stone, B. A.; McDougall, B. M.; Bacic, A.; Martin, K. L.; Brownlee, R. T. C.; Chai, E.; Seviour, R. J.; Carbohydr. Res. 2001, 331, 163.

27. Bao, X-F.; Fang,J.; Li, X-Y.; Biosci., Biotechnol., Biochem. 2001, 65, 2384.

28. Lowe, E.; Rice, P.; Há, T.; Li, C.; Kelley, J.; Ensley, H.; Lopez-Perez, J.; Kalbfleisch, J.; Lowman, D.; Margl, P.; Browder, W.; Williams, D. A.; Microbes and Infection 2001, 3, 789.

29. Agrawal, P. K.; Phytochemistry 1992, 31, 3307. 


\section{PURIFICAÇÃO E CARACTERIZAÇÃO DE UMA GENTIOEXAOSE OBTIDA DE BOTRIOSFERANA POR HIDRÓLISE ÁCIDA PARCIAL}

Iara Ribeiro Silva, Nilson Kobori Monteiro, Paula Felippe Martinez, Nataly Lino Izeli, Ana Flora Dalberto Vasconcelos, Marilsa de Stefani Cardoso e Maria de Lourdes Corradi da Silva*

Departamento de Física, Química e Biologia, Faculdade de Ciências e Tecnologia, Universidade Estadual Paulista Júlio de Mesquita Filho, CP 467, 19060-900 Presidente Prudente - SP, Brasil

Aneli de Melo Barbosa

Departamento de Bioquímica e Biotecnologia, Centro de Ciências Exatas, Universidade Estadual de Londrina, CP 6001,

86051-990 Londrina - PR, Brasil

Robert F. H. Dekker

Universidad de Castilla-La Mancha - IRICA, 13071 Ciudad Real, España

Gil Valdo José da Silva e Luís Alberto Beraldo de Moraes

Departamento de Química, Faculdade de Filosofia, Ciências e Letras de Ribeirão Preto, Universidade de São Paulo, 14040-901 Ribeirão Preto - SP, Brasil
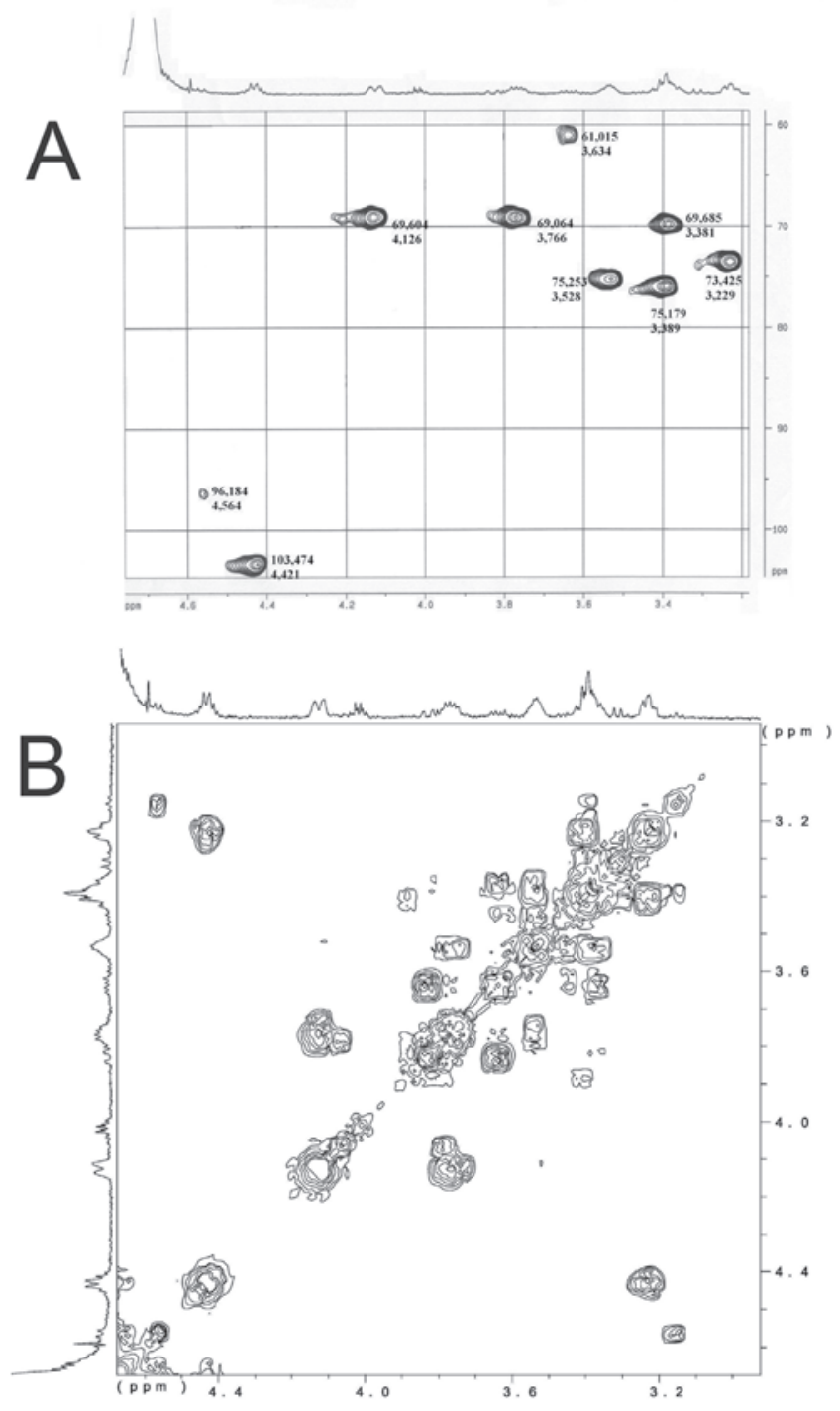

Figura 1S. Espectros de ressonância magnética nuclear em $500 \mathrm{MHz}$ do componente com $R_{T} 16,76$ min. (A) $\mathrm{HMQC}\left({ }^{1} \mathrm{H}-{ }^{13} \mathrm{C}\right)$ e $(\mathrm{B}) \mathrm{COSY}\left({ }^{1} \mathrm{H}-{ }^{1} \mathrm{H}\right)$ 

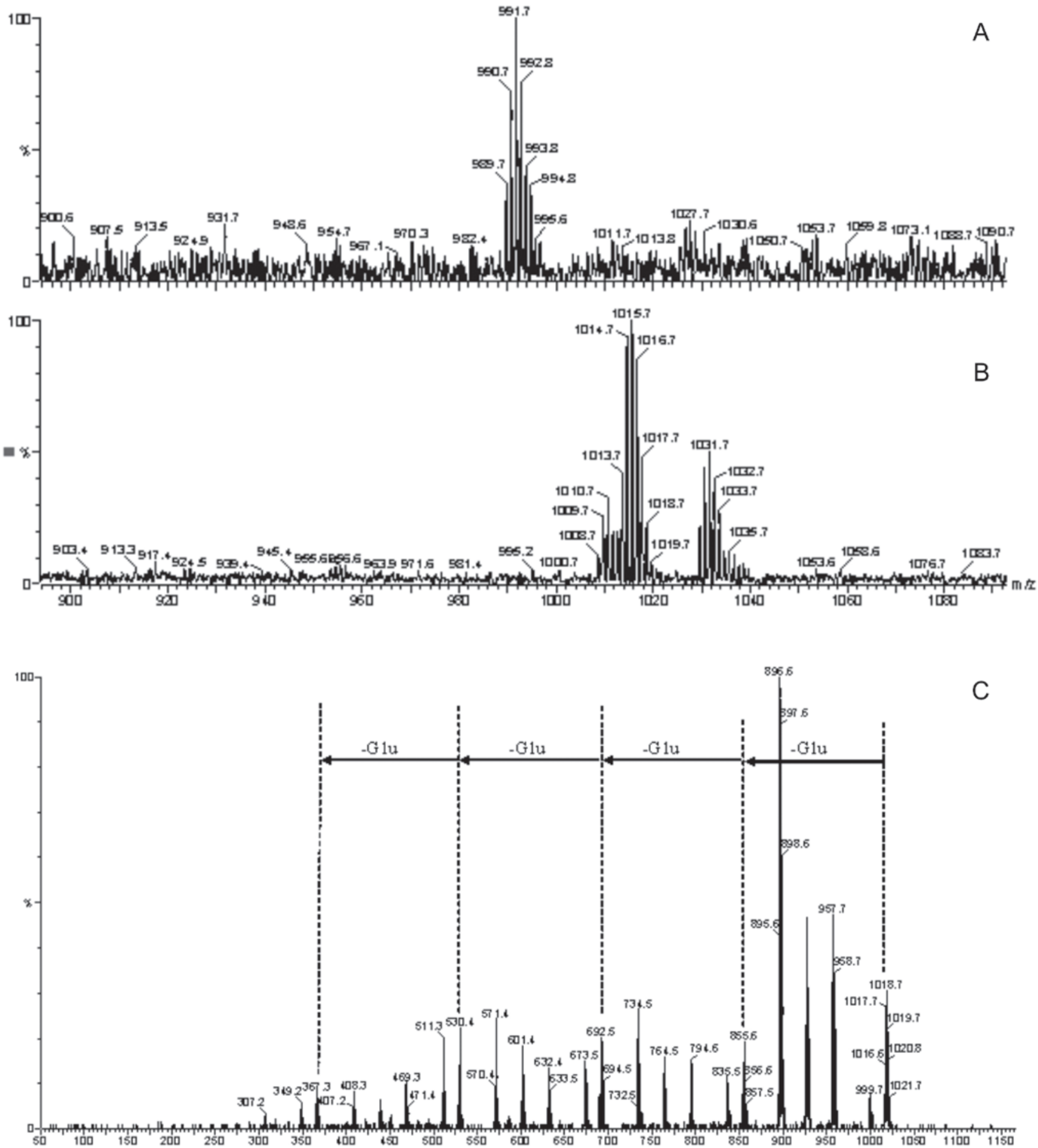

Figura $2 S$. Espectros de massa com ionização por electrospray. A - modo negativo, B- modo positivo, $C$ - espectro de fragmentação 